\title{
New solution for transport and industrial noise protection through reflective noise barriers
}

\author{
Ivan $\mathrm{Kralov}^{1, *}$ \\ ${ }^{1}$ Technical University - Sofia, 8 Kliment Ohridski Blvd., 1000 Sofia, Bulgaria
}

\begin{abstract}
A new solution for protection of transportation and industrial noise through reflective noise barriers is proposed and investigated in this study. The new solution combines the advantages of the known barriers and has its own advantages in addition. The preliminary results show a very good level of noise reduction for this type of barriers.
\end{abstract}

\section{Introduction}

Acoustic noise reduction is a very important process due to its direct impact on the environment. The main problem in this area is the noise created by the specific functional processes of the machines. In most of these cases the typical solution is to reduce its propagation by different types of barriers, absorbers or active noise reduction solutions. The topicality of the problem mentioned above is proven by the enormous number of recent research work and publications $[10,11,12,13]$. In many cases, expensive and sophisticated design and technological solutions have to be created in order to meet the noise reduction standards. The methods for achieving noise reduction are mainly divided into passive, active and combined. Passive methods generally block the spreading of sound waves and decrease the noise levels by reflecting and absorbing their energy $[1,2,3,10,11,12,13]$. On the other hand, active methods input additional energy that is used to eliminate the acoustic energy of the source by creating significant noise background, propagation of sound waves in the opposite phase etc. [1, 2, 13]. Combined methods include both of the previously mentioned ones.

The subject of the present research is part of the passive methods of noise reduction because of their low cost, manufacturing technology, installation and usage, as well as the opportunity for high feasibility in industry. The main principle in the considered patent solutions is reflecting the sound waves, generated from the source, using profile barriers. In most of these cases there is also a considerable absorption of the barrier.

A classical example of a reflective barrier is described in Patent № UD 96/16230 - 30.05.1996 [4]. Convex arcshaped reflecting profiles are fixed to the vertical concrete columns as shown on Fig. 1. Part of the incoming sound waves are reflected by the barrier and these waves interfere with the original ones close to the barrier surface performing noise reduction. Another part of the source sound waves is absorbed by the barrier while a third part passes through it. Some of the advantages of this solution are: a wide area of application, a stable construction consisting of one type of reflecting elements and relatively low cost of production. Different columns in case of different heights of the barrier, expensive transportation and assembly are the main disadvantages. From an acoustic point of view, the edges of the profiles joints are concentrators of source and reflected sound energy and in this capacity they could be treated as a secondary line source of sound. In this way the noise level near the barrier could be significant.

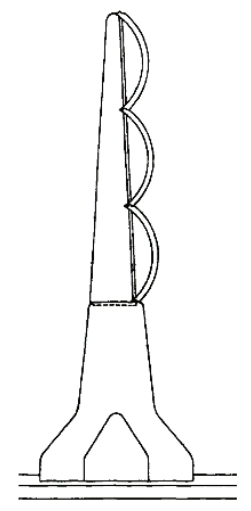

Fig. 1. Fixture of convex arc-shaped reflecting profiles to vertical concrete column.

Patents №№ US № 6305492 B1 / 23.10.2001 [5] and US 7789193 B2 / 07.09.2010 [6] are similar to the above described ones, but their reflecting surfaces have concave arc profiles - Fig. 2 or hexagonal concave profiles - Fig. 3 . The main advantage in both cases is reduction through interference of the source and reflected waves near the barriers. The disadvantages are expensive production, transportation and high assembly costs especially for the second solution.

The noise barrier under Patent № AT 513615 A4 2014-06-15 [7] is built from elastic elements made from used car tires - Fig. 4. They are assembled to be able to make small vibrations. This construction has a few

\footnotetext{
* Corresponding author: kralov@tu-sofia.bg
} 
advantages: use of materials normally considered waste (used car tires), higher degree of absorption due to internal damping in the rubber during vibrations and reduction through interference of the source and reflected waves near to barriers. Some problems of this solution are connected with how they look as well as their low climate resistance.

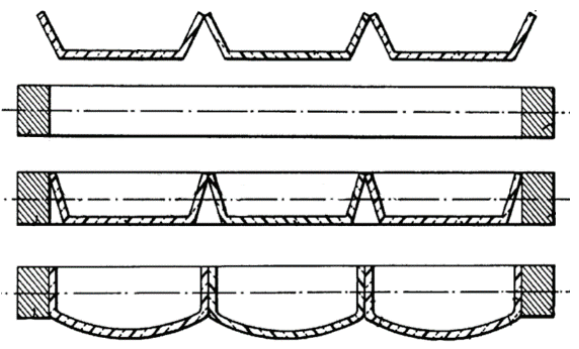

Fig. 2. Reflecting surfaces with concave and arc profiles.

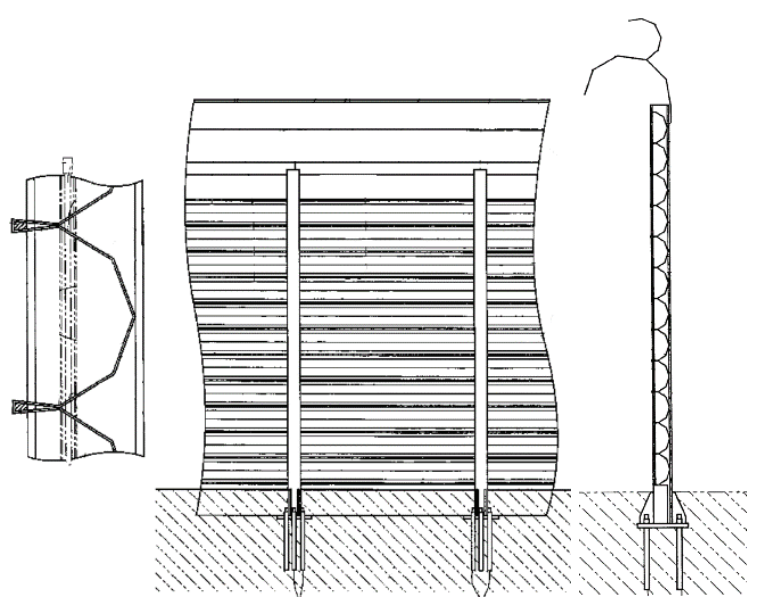

Fig. 3. Reflective barriers with hexagonal concave profiles.

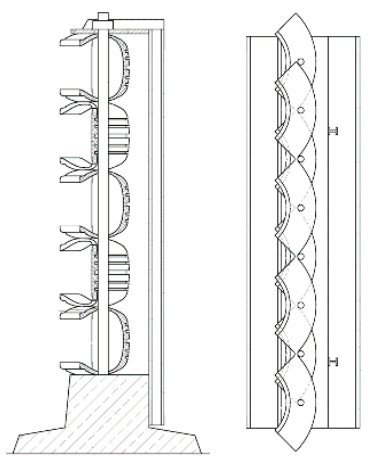

Fig. 4. Barriers with elastic elements made from used car tires.

Patent solutions №№ 89203035.4 / 29.11.1989 [8] (Fig. 5) and DE 4220547 A1 / 07.01.1993 [9] (Fig. 6) cover preliminarily automotive and railway transportation noise insulation. A specific characteristic of the first one is the possibility to change the angle of the reflecting surface. This is a simple construction, but very expensive to assemble and maintain. The second solution has a construction which allows to add heavy absorbing material layers to the barrier. This is also a simple, relatively cheap and noise-reduction effective solution. The main disadvantage is that it is not applicable in the open air due to the absence of climate resistance /rain, snow etc./ of the absorbing materials.

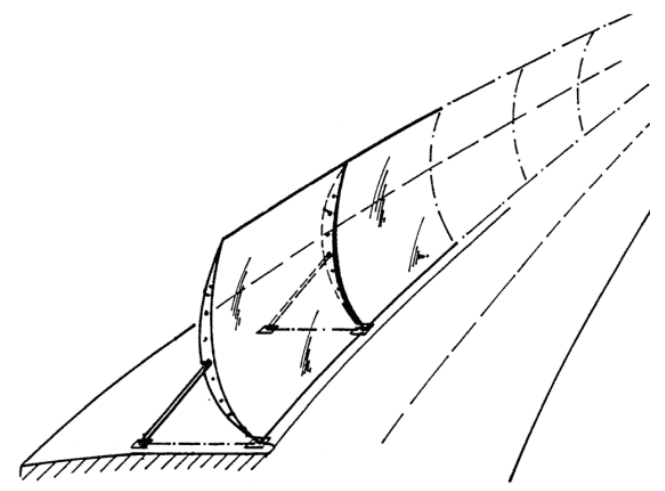

Fig. 5. Preliminarily automotive and railway transport noise insulation.
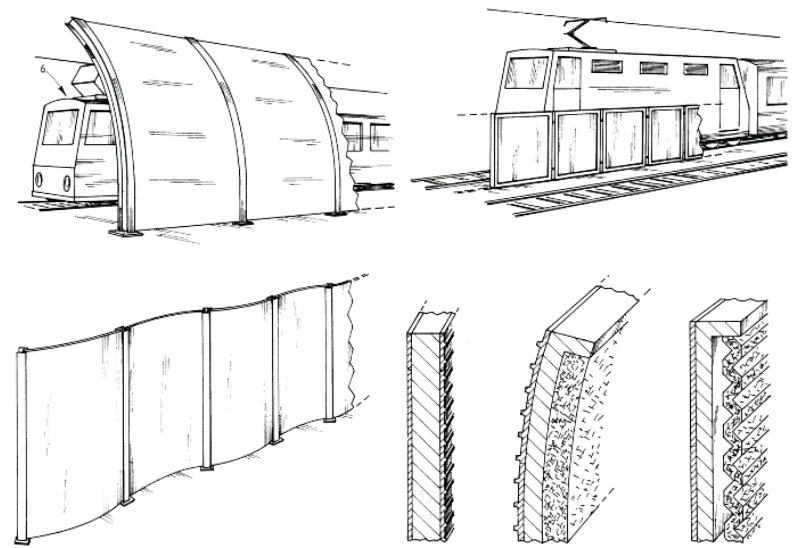

Fig. 6. Preliminarily automotive and railway transportation noise insulation.

Nowadays, barriers, similar to patent solution 102014 2017 767.7 / 10.03.2016. [10] (Fig. 7) are used widely. Barriers are made of long metal panels, lined one over the other and fixed between vertical metal columns. Some variants of the solution are perforated panels, or/and presence of absorbing material inside. This type of construction is effective, easy to transport and assemble, climate and fire resistant, but it has very high production cosst. This costs are due to the used material - aluminium, the expensive technology for mechanical perforation of the panel, as well as the assembly of the absorbing material.
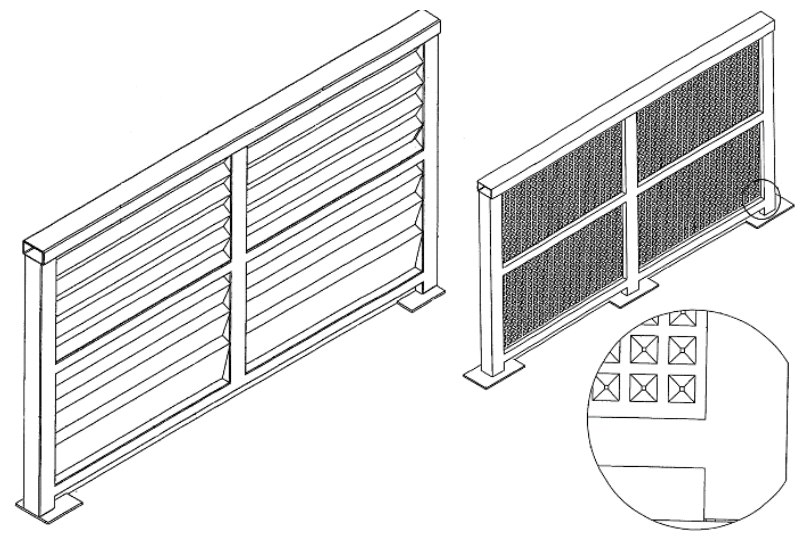

Fig. 7. Commonly used preliminarily automotive and railway transportation noise insulation. 
On the base of the analysis of the above described main patent solutions for passive noise reduction barriers, the following conclusions could be made:

The great number of patent solutions for passive noise reduction barriers are based on the use of different profile reflecting surfaces.

Some noise barriers are made by assembly elastic elements in the construction. Their small vibrations excited by the sound energy increase the degree of absorption. In a few cases these elements are industrial waste and the ecological effect is a bonus.

The efficiency of the passive noise barriers is increased by adding additional layers of highly absorbing material.

A significant part of the proposed and used solutions for passive noise reduction have good level of insulation, but their production, transportation, assembly and maintenance costs are very high.

Taking into consideration the above conclusions, the aim of this study is defined as the synthesis of a new passive noise barrier construction, which has a minimum of the described disadvantages. To reach the final result three main problems need to be solved: define the design principles, design the construction and determine and verify its main acoustic parameters.

\section{New construction of a noise insulating panel and barrier}

As a result of the analysis above the following principles for the new barrier synthesis are used:

- passive noise barrier for transportation and industrial noise;

- level of noise reduction equal or better to that of the existing barriers;

- relatively low costs of production, transportation, assembly and maintenance;

- relatively high level of climate change resistance, windproof and fire-proof construction.

As a result of the synthesis the new noise insulating panel and barrier constructions are shown on Fig. 8. Noise insulating panels are made from non-metal materials. They are one of many horizontal channels arranged one over the other, and all their concave arc profiles have one and the same direction - to the sound source waves. The essential characteristic of the construction is that the concave profile is exactly semi-cylindrical and the border area between two neighbor channels is a sharp edge. These channels are arranged into H-shaped metal columns fixed to the ground. The channels are fixed vertically to the columns through non-metal semi-disks of external diameter, equal to the internal diameter of the channels. The semi-disks are fixed to the columns by bolt or stud connection.

The main expected effect of the new solution is that the reflection of the sound waves and the interference between the source and reflected ones is close to the barrier. In this way the noise reduction by converting sound energy into heat will not affect the noise sources. The degree of reflection depends mainly on the channel material, sound waves frequency and acoustic pressure. An important parameter of the barrier construction is the absence of reflecting surface, perpendicular to the source sound waves. The exact semi-cylindrical profile of the channels focuses reflected waves to its geometrical center. This reduces the area of interference and noise reduction.

Geometrical parameters of the barrier can be designed for specific cases to achive better performance. The width of the profile and the radius of the channel could by varied according to the sound power and frequency.

The modular construction has a lot of advantages. First, the non-metal channels are technologically easy to produce, for example by extrusion. The uniform elements allow for big series production. This reduces production costs. Second, the elements are easy to be transported, assembled and maintained. Assembly does not require neither special machines nor expensive equipment, and there is no need for the working team to posess any special skills. Third, the modular construction makes it possible to vary easily the height of the barriers.

By using appropriate materials some additional requirements like climate resistance and fire-proofing could be fulfilled. Other common requirements like construction stiffness, wind resistance etc. have to be designed using the common rules.

The wide area of application of the new barriers is an additional advantage. They could be used for noise insulation of automotive and railway roads, for industrial sites, for buildings, etc.

\section{Numerical investigation procedure verification}

Comsol multiphysics ${ }^{\circledR}$ acoustic module will be used for numerical investigation of the new barrier noise reduction. Before this step, an experimental setup as well as its numerical model were created to verify the numerical procedure used.
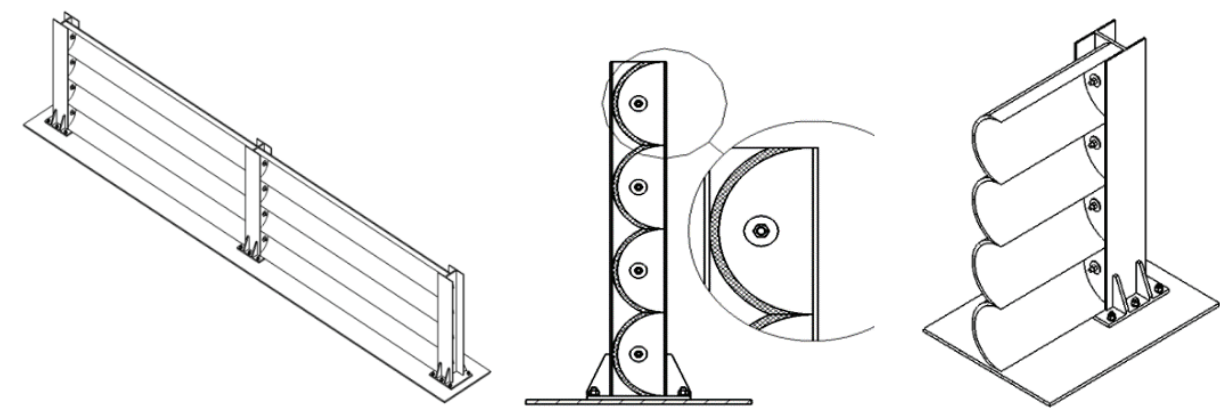

Fig. 8. New noise insulating panel and barrier. 
This experimental setup and the testing procedure of the absorber are shown in Fig. 9. The noise, generated by the noise generator and powered by the amplifier, is emitted in the $30 \mathrm{~dB}$ acoustic chamber. The front side of the chamber is open. At a distance of $0,5 \mathrm{~m}$ the microphone registers the noise levels. In this way the noise levels of the source without a barrier are recorded. After the first experiment, the testing absorber is attached to the chamber front side (Fig. 9).

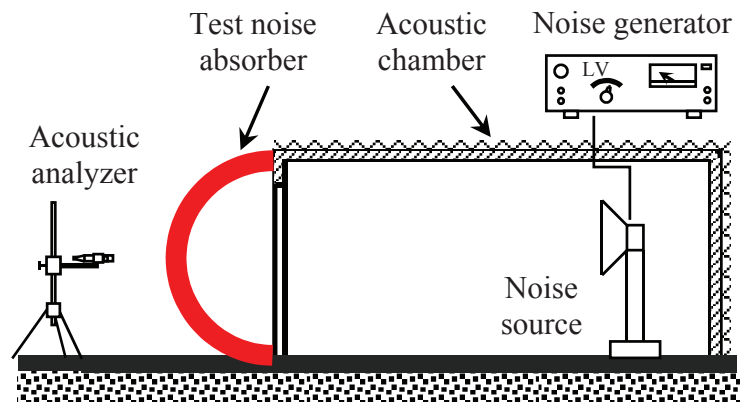

Fig. 9: Scheme of the noise absorber testing procedure.

The absorber is made of high density polyvinile (HDPV) material and is shaped as a semi-circular tube with a diameter of $500 \mathrm{~mm}$ and a width of $10 \mathrm{~mm}$. All holes are insulated and covered by high-level absorbing materials. The same noise levels are generated by the noise-source equipment. The microphone registers the noise levels at the same position after the new absorber is applied. A XL2, class 1, certified acoustic analyzer produced by NTi company is used. All the results are collected according to the standards and legislations concerning calibration and testing procedures, as well as measurement error evaluation.

The numerical model of the above described experimental setup is investigated using the $\mathrm{Comsol}^{\circledR}$ software. Noise levels in decibels $(\mathrm{dB})$ are measured in both procedures.

The noise reduction level from experimental tests $\Delta L_{E i I}$ for $i$-th frequency are defined as the difference between the measured noise level without absorber $L_{E, i}$ and measured noise level without absorber $L_{E A, i}$ :

$$
\Delta L_{E i I}=L_{E, i}-L_{E, I} .
$$

The noise reduction level from numerical tests $\Delta L_{N i I}$ for $i$-th frequency are defined as the difference between the measured noise level without absorber $L_{N, i}$ and measured noise level without absorber $L_{N A, i}$ :

$$
\Delta L_{N i I}=L_{N, i}-L_{N, I}
$$

The results about noise reduction with both procedures are given in Table 1.

Table 1. Noise level reduction for test sound barrier experimental and numerical results

\begin{tabular}{|c|c|c|}
\hline $\mathrm{Hz}$ & $\Delta L_{E i I}, \mathrm{~dB}$ & $\Delta L_{N i I}, \mathrm{~dB}$ \\
\hline 125 & 4,7 & 4,9 \\
\hline 250 & 8,2 & 9,7 \\
\hline 500 & 15,6 & 16,9 \\
\hline 1000 & 10,4 & 12,3 \\
\hline 2000 & 21,6 & 23,3 \\
\hline
\end{tabular}

There is a very small difference for each frequency between the two procedures. On the basis of these results, it could be stated that the used numerical procedure is appropriate for the planned investigation.

\section{Noise reduction efficiency of the new barrier}

The main studied parameter of the newly designed noise insulating barrier is the degree of noise reduction.

First, using the advantages of the advanced digital technologies, a numerical model of the barrier was created. For meshing, defining of the boundary conditions and other parameters, software guidelines and good practices were used.

Next, a numerical simulation of the noise radiation and reduction of the model was done. Commercial software for CAD and for Acoustic analysis were used.

Parameters of the investigated model are:

- Channel material - high density PVC /HDPVC/;

- Width of the channel - $20 \mathrm{~mm}$;

- Diameter of the channel - $600 \mathrm{~mm}$;

- Height of the barrier - 4,2 m;

- Height of the noise source $-1 \mathrm{~m}$;

- Type of the noise waves - spherical;

- Horizontal distance between the source and front of the barrier - 7,5 m;

- Height of the "microphone" position - 1,5 m;

- Horizontal distance between the "microphone" position and the back of the barrier $-5 \mathrm{~m}$.

To compare the results of this study with similar ones, most of the parameters are chosen to replay the standard requirements or to correspond the data from other research works.

The environment around the barrier is air of density of $1,19 \mathrm{~kg} / \mathrm{m}^{3}$ and speed of sound of $343 \mathrm{~m} / \mathrm{s}$.

The barrier material is HDPE with $970 \mathrm{~kg} / \mathrm{m}^{3}$ density, $2310 \mathrm{~m} / \mathrm{s}$ speed of sound, 0,40-0,45 Poisson's ratio, and $1,035 \mathrm{~N} / \mathrm{mm}^{2}$ Young's modulus.

Noise reduction is measured for the central frequencies of the $1 / 3$ octave bands: $125 \mathrm{~Hz}, 250 \mathrm{~Hz}$, $500 \mathrm{~Hz}, 1000 \mathrm{~Hz}, 2000 \mathrm{~Hz}$. Formula 2 is used for evaluation of the degree of noise reduction. A graphical representation of the results for $250 \mathrm{~Hz}$ and $1000 \mathrm{~Hz}$ is given in Fig. 10 and Fig. 11. Results from the numerical simulations are presented in Table 2.

The maximal level of noise reduction for the studied frequencies is $16,1 \mathrm{~dB}$ at $2000 \mathrm{~Hz}$.

On the basis of the obtained results about the level of noise reduction, with the new barrier, it could be stated that:

These results show very good noise reduction of the new noise insulating barrier in general.

There is a big difference in noise level reduction for different frequencies for the studied barrier. It could be the result of resonant modes of the barrier in some frequencies, as well as of the different "noise shadow" at different frequencies.

The minimal level of noise reduction for the studied frequencies is $6 \mathrm{~dB}$ at $500 \mathrm{~Hz}$. 


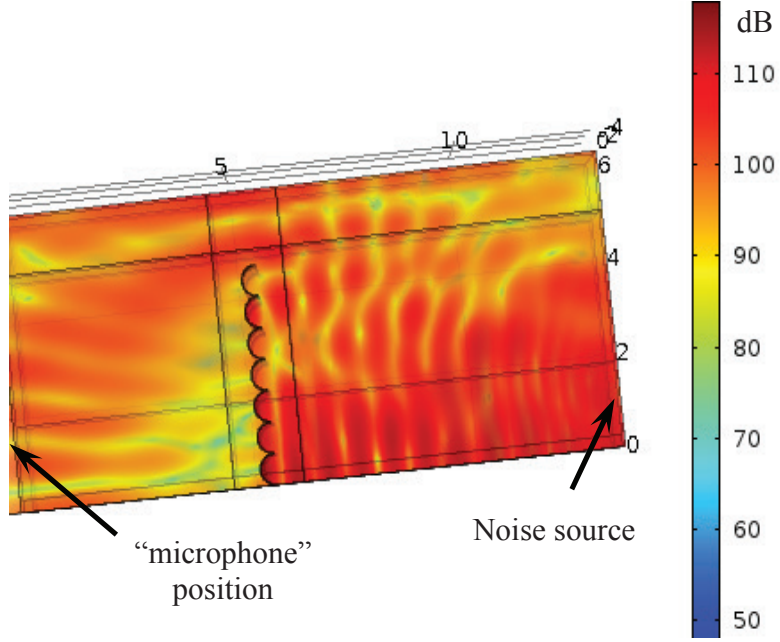

Fig. 10. Numerical results for $250 \mathrm{~Hz}$.

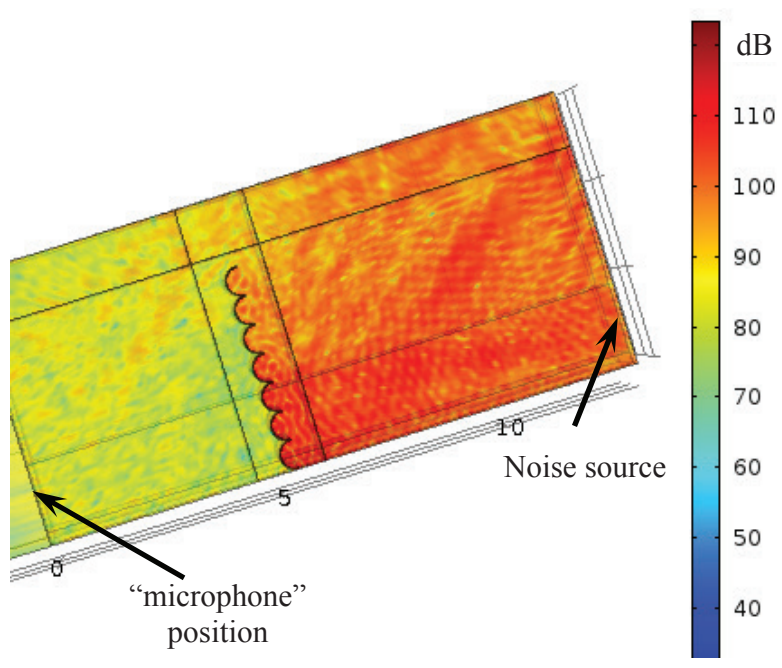

Fig. 11. Numerical results for $1000 \mathrm{~Hz}$.

Table 2. Noise level reduction with the new sound barrier numerical results

\begin{tabular}{|c|c|}
\hline $\mathrm{Hz}$ & $\Delta L_{N i I}, \mathrm{~dB}$ \\
\hline 125 & 10,5 \\
\hline 250 & 13,0 \\
\hline 500 & 6,0 \\
\hline 1000 & 16,0 \\
\hline 2000 & 16,1 \\
\hline
\end{tabular}

The next step in the investigation will be to find the influence of some geometrical parameters upon the noise reduction efficiency of the barrier.

\section{Conclusion}

A new solution for insulation of transportation and industrial noise by means of reflective noise barriers has been proposed and investigated in this study. It combines the main advantages of currently used barriers and it also has additional ones. Numerical simulation shows high degree of noise reduction. To improve the efficiency of noise reduction a further investigation of the influence of the barrier parameters upon the frequency absorption response has to be made.

\section{References}

1. S. Banov, I. Kralov, Noise in Transportation Technics, TU-Sofia, Sofia, (in Bulgarian), (2003)

2. Nikolov, N., D. Benov, I. Shubin, Acoustic Design of Transport Noise Insulating Barriers, ACMO Academic Press, Sofia, (in Bulgarian), (2014)

3. Patent № UD 96/16230 - 30.05.1996.

4. Patent № US № 6305492 B1 / 23.10.2001.

5. Patent № US 7789193 B2 / 07.09.2010.

6. Patent № AT 513615 A4 2014-06-15.

7. Patent № 89203035.4 / 29.11.1989.

8. Patent № DE 4220547 A1 / 07.01.1993.

9. Patent № 1020142017767.7 / 10.03.2016.

10. I. Kralov, S. Terzieva, I. Ignatov, Analysis of methods and MEMS for Acoustic Energy Harvesting with Application in Railway Noise Reduction, Proceedings of the MECAHITECH'11, Bucharest, (2011).

11. I. Kralov, P. Sinapov, K. Nedelchev, I. Ignatov, Friction Induced Rail Vibrations, AIP, 1497, 19-25, (2012)

12. J. Trevor, P. D'Antonio, Acoustic Absorbers and Diffusers, Taylor \& Francis, (2009)

13. D. J. Thompson, Railway Noise and Vibration, Elsevier, (2009) 